\title{
Ultraviolet Inactivation of Chikungunya Virus
}

\author{
Asha Maria Mathew Amol Baburao Mun Anukumar Balakrishnan \\ Kerala Unit, National Institute of Virology, T.D Medical College Hospital, Alappuzha, India
}

\section{Keywords}

Ultraviolet inactivation · Chikungunya virus · CL-1000

crosslinker

\section{Abstract}

Objective: Chikungunya virus (CHIKV) is a rapidly emerging arbovirus causing millions of infections in more than 40 countries. CHIKV is typically a biosafety level 3 pathogen in many countries and handling of CHIKV requires a high standard of laboratory safety settings. Many studies require the whole virus to be handled in a biosafety level 2 setting. A potential solution for managing this problem is pathogen inactivation without affecting its antigenicity. In the present study, we attempted to inactivate CHIKV by ultraviolet (UV) irradiation. Methods: Different UV doses were used to inactivate CHIKV. The replication status of the inactivated virus was verified in cell lines. Western blot, electron microscopy, and immune fluorescence assay were used, respectively, to view the antigenicity, structural integrity, and entry of the virus into cell lines. Results: The inactivation was complete when a UV dose of $0.09 \mathrm{~J} / \mathrm{cm}^{2}$ for $3 \times 30 \mathrm{~s}$ was used and no change in antigenicity and integrity was observed. Conclu-

\section{KARGER}

(c) 2018 S. Karger AG, Basel

E-Mail karger@karger.com

www.karger.com/int sions: The study concludes that the UV-inactivated virus is antigenically stable and could be used in biosafety level 2 settings for different experiments.

(c) 2018 S. Karger AG, Basel

\section{Introduction}

Chikungunya virus (CHIKV) infection has been reported in more than 40 countries. This pathogen is considered a category $\mathrm{C}$ priority pathogen by the National Institute of Allergy and Infectious Disease (NIAID) [1]. CHIKV is a small, enveloped, Old World RNA virus in the family Togaviridae (Alphavirus genus). The symptoms of CHIKV infection include fever, arthralgia (severe joint pain), and rash $[1,2]$. The joint pain may persist for months or even years in many of those infected [3]. CHIKV is transmitted by mosquitoes, typically the Aedes species, and replicate in both arthropod and vertebrate hosts [2]. The CHIKV genome is composed of a single strand of positive-sense RNA about $11.8 \mathrm{~kb}$ in length. The RNA encodes 4 nonstructural proteins (nsP1, nsP2, nsP3, and nsP4) and 5 structural proteins (capsid, E3, E2, 6K 
and E1). The nonstructural proteins are involved in virus replication and pathogenesis, and structural protein makes up the virion $[4,5]$.

CHIKV has a high laboratory safety requirement. As CHIKV disease represents a continuing threat to the healthcare and the medical world, safer and more costeffective diagnostic approaches that can be practiced in a low biosafety level laboratory setup that also ensures the rapid detection of CHIKV infection is desirable. Many applications, such as PCR and antigens for capture ELISA, require the whole virus. Most laboratories depend on a commercial source for the inactivated virus [6], which is not economical. Having a simple and economical inactivation method would be a potential solution for this problem.

Researchers from different parts of the world have developed many methods to inactivate viruses, including heat inactivation, ionizing radiation, $\mathrm{pH}$ inactivation, chemical inactivation, and ultraviolet (UV) inactivation [7-13]. Chemical inactivation induces extensive interaction and the cross-linking of viral proteins, and thus it can alter the conformational surface epitopes. Heat inactivation is a simple and cost-effective method, but it has been shown that the prolonged incubation time required for inactivation can cause structural changes in viral proteins and degradation of viral genetic material [12].

UV irradiation has been known as an effective method to inactivate viruses for a long time and it preserves the integrity of the immunological epitopes [7]. The absorption of UV causes photochemical damage in viruses as UV induces adjacent pyrimidine nucleotide dimerization. This can create mutations, finally inhibiting virus replication. As RNA viruses lack repair mechanisms, they can be easily inactivated by UV [8]. It has been proven experimentally that ssRNA are more sensitive against UV irradiation. Studies suggest that ssRNA viruses like Caliciviridae, Orthomyxoviridae, Picornaviridae, and Togaviridae are highly sensitive to UV [7-9].

A number of studies have been reported on the inactivation of CHIKV using chemicals like formalin, $\beta$-propiolactone, 1,5-iodonapthylazide (INA), and binary ethyleneimine for vaccine preparations [13-18]. However, these chemicals are expensive, toxic, and the virus inactivation process using them can alter the antigenicity of the virus. UV inactivation is a comparatively simple and less expensive method. A combination of UV-inactivated and formalin-treated CHIKV was shown to be effective in stimulating monkeys to produce neutralizing antibodies, and found to be better than formalin-treated CHIKV vaccine by some researchers [16]. Sharma et al. [15] used a combination of UV and INA to inactivate VEEV and the same protocol was extrapolated to inactivate CHIKV [18]. Upon reexamination of the experimental details of the previous CHIKV UV inactivation protocols available, we found that the applied UV doses and exposure time was high and there were no systematic studies on the inactivation of CHIKV using UV light alone. Here, we determined to study the inactivation kinetics of CHIKV using UV light.

\section{Materials and Methods}

\section{Virus and Cell Lines}

The CHIKV Asian isolate (IND-63-WB1) was kindly provided by Dr. A.B. Sudeep, NIV, Pune, India. Vero and RD cell lines were obtained from cell repository, NCCS, Pune, India.

The CHIKV was propagated in Vero cells (African Green Monkey kidney, P-36) maintained in Dulbecco's modified Eagle medium (DMEM; HiMedia, India) supplemented with $2 \%$ fetal bovine serum (FBS; Gibco, Invitrogen) at $37^{\circ} \mathrm{C}$ in an atmosphere of $5 \% \mathrm{CO}_{2}$. The virus titer was determined by standard plaque assay.

\section{Plaque Assay}

Virus titer in the culture supernatant was quantitated by plaque assay. Briefly, a 24-well tissue culture plate containing a confluent monolayer of Vero cells was infected with 10-fold dilutions of CHIKV. Following incubation for $1 \mathrm{~h}$ at room temperature (RT), the cells were washed and overlaid with a $1.5 \%$ final concentration of carboxy methyl cellulose in DMEM with $2 \%$ FBS. The plate was incubated for $48 \mathrm{~h}$ at $37^{\circ} \mathrm{C}$ with $5 \% \mathrm{CO}_{2}$. The plate was then stained with $0.1 \%$ crystal violet in formal saline. The plaques were counted and expressed as plaque- forming units per $\mathrm{mL}(\mathrm{pfu} / \mathrm{mL})$.

\section{UV Inactivation of CHIKV}

UV inactivation of the virus was carried out using a CL1000 UV crosslinker (Analytik, Jena, Germany). The culture supernatant (2 $\times 10^{7} \mathrm{pfu} / \mathrm{mL}$ ) was placed in a $35-\mathrm{mm}$-diameter petri dish with a depth of $2 \mathrm{~mm}$. The distance between the UV light and petri dish was about $12 \mathrm{~cm}$. The supernatant was treated with different doses of UV light ranging from 0.02 to $0.11 \mathrm{~J} / \mathrm{cm}^{2}$. The respective dose was given 3 times for $30 \mathrm{~s}$ to achieve maximum inactivation. The inactivation efficiency was confirmed by 3 blind passages in Vero cells. The presence of a cytopathic effect (CPE) indicated the incomplete or partial inactivation of the virus.

\section{SDS-PAGE and Western Blot}

The antigenicity of the inactivated CHIKV was confirmed by Western blot using anti-CHIKV monoclonal antibodies. The inactivated virus supernatants $(15 \mu \mathrm{L})$ were loaded onto $10 \%$ polyacrylamide gel. The viral proteins were separated by electrophoresis. The separated proteins were transferred onto a PVDF membrane (GE Healthcare, Piscataway, NJ, USA) using a semidry transfer unit (TE 70 SemiPhor transfer unit, Amersham Bioscience, CA, USA). The membrane was washed with wash buffer (Tris-buffered saline containing $0.1 \%$ Tween-20). The membrane was then blocked with 5\% skimmed milk (Cell Signaling 
Technology) in wash buffer for $1 \mathrm{~h}$ at RT. The membrane was washed and subsequently incubated for $1 \mathrm{~h}$ at RT with mouse CHIKV monoclonal antibodies (capsid/E2) diluted in 3\% BSA in wash buffer. The membrane was then washed 3 times and incubated with horseradish peroxidase-conjugated anti-mouse antibody (Sigma) for $45 \mathrm{~min}$ at RT. The proteins were detected using ECL reagent (GE Healthcare) according to the manufacturer's instructions.

\section{Electron Microscopy}

The integrity of the inactivated virus was confirmed by transmission electron microscopy. The purified virus was adsorbed on a copper grid and negatively stained with phosphotungstic acid. The stained grid was imaged using a $120 \mathrm{KeV}$ operating voltage in a Tecnai-12 Biotwin transmission electron microscope (FEI Co., Eindhoven, The Netherlands).

\section{Immunofluorescence Assay}

The attachment, entry, and replication status of inactivated virus to the cells was studied by immunofluorescence assay. A confluent monolayer of RD cells (human rhabdomyosarcoma, P-23) in a 24-well plate (Nunc, Thermo Scientific) was treated with 10 MOI (multiplicity of infection) of inactivated CHIKV. Cells infected with live virus were kept as a positive control. After $24 \mathrm{~h}$ postinfection, the cells were fixed with $4 \%$ paraformaldehyde in PBS and permeabilized with $0.1 \%$ Triton $\times 100$ in PBS for 10 min. After blocking the cells with $3 \%$ BSA in PBS, the cells were incubated for $1 \mathrm{~h}$ at $37^{\circ}$ with an in house-generated anti-CHIKV mouse monoclonal antibody (1:50). The cells were washed and incubated with Alexa 488-conjugated anti-mouse antibody (R37120, Invitrogen) for $45 \mathrm{~min}$ at $37^{\circ} \mathrm{C}$. The cells were counterstained with DAPI (Sigma) for $2 \mathrm{~min}$. The cells were observed under a FLoid cell imaging station (Thermo Fisher Scientific) or Nikon Ti eclipse inverted microscope.

\section{Results}

\section{Inactivation of $\mathrm{CHIKV}$}

Different doses of UV starting from 0.02 to $0.11 \mathrm{~J} / \mathrm{cm}^{2}$ were tried and the complete inactivation of the virus was observed at a UV dose of $0.09 \mathrm{~J} / \mathrm{cm}^{2}$ for $3 \times 30 \mathrm{~s}$ (Fig. 1). At this energy dose of UV, no CPE was noticed even after 3 blind passages in Vero cells.

\section{Western Blot and Electron Microscopy}

Western blot analysis showed that different bands, corresponding to the live viral proteins, were also present in the UV-inactivated virus. A $50-\mathrm{kDa}$ band corresponding to the $\mathrm{E} 2$ protein and 1 band around $30 \mathrm{kDa}$ corresponding to the capsid protein were present in both the live and inactivated virus. These results suggest that the envelope protein and capsid protein were capable of binding to antibodies, just like the native CHIKV (Fig. 2). The electron micrograph showed

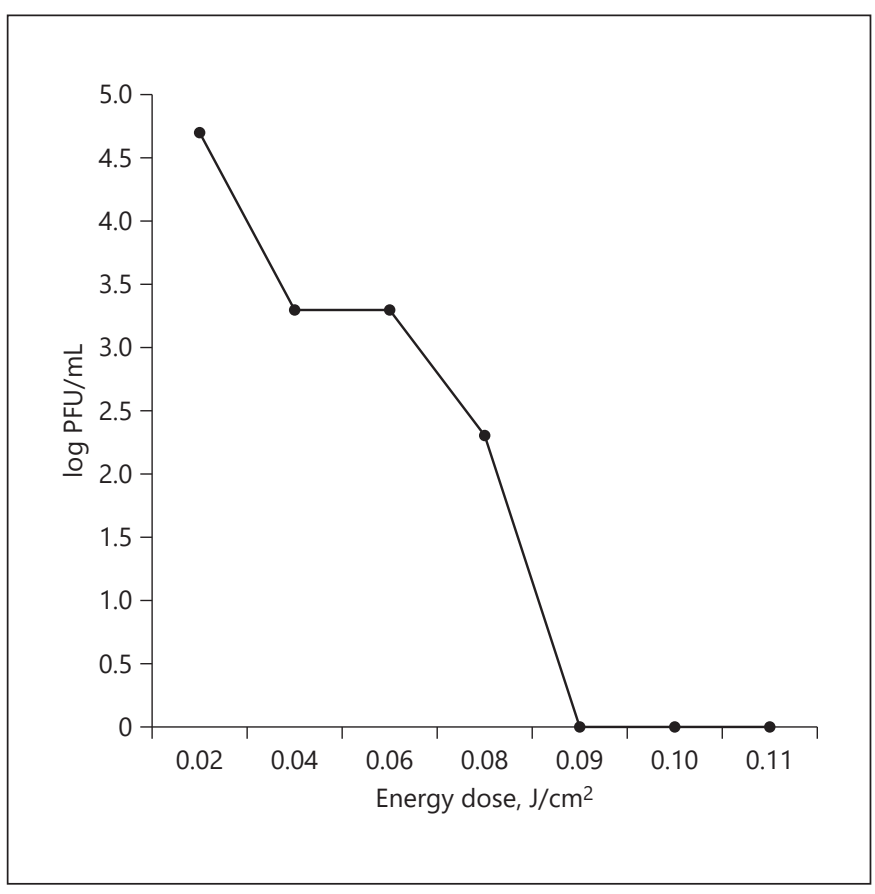

Fig. 1. Dose-dependent inactivation of CHIKV by UV irradiation. The CHIKV $\left(2 \times 10^{7} \mathrm{pfu} / \mathrm{mL}\right)$ was inactivated by different doses of UV using a UV crosslinker. The inactivation was confirmed by plaque assay.

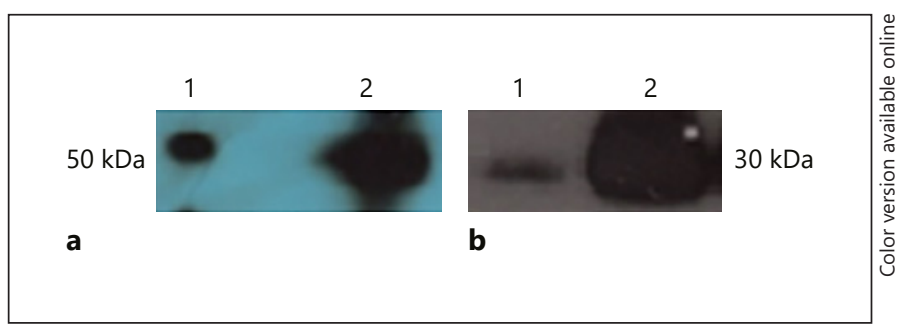

Fig. 2. Western blot analysis of UV-inactivated CHIKV probed with monoclonal E2 (a) or capsid antibodies (b). CHIKV control (1) and UV-inactivated CHIKV (2; dose $\left.0.09 \mathrm{~J} / \mathrm{cm}^{2}\right)$ showing band sizes around 50 and $30 \mathrm{kDa}$ corresponding to $\mathrm{E} 2$ and capsid, respectively.

spherical, intact particles of $65-70 \mathrm{~nm}$, similar to live CHIKV (Fig. 3).

\section{Replication, Binding, and Entry of UV-Inactivated CHIKV}

The attachment, entry, and replication status of UVinactivated virus was checked by immunofluorescence as- 
Fig. 3. Electron micrograph of CHIKV (a) and UV-inactivated CHIKV (b) showing particles of approximately $65-70 \mathrm{~nm}$.
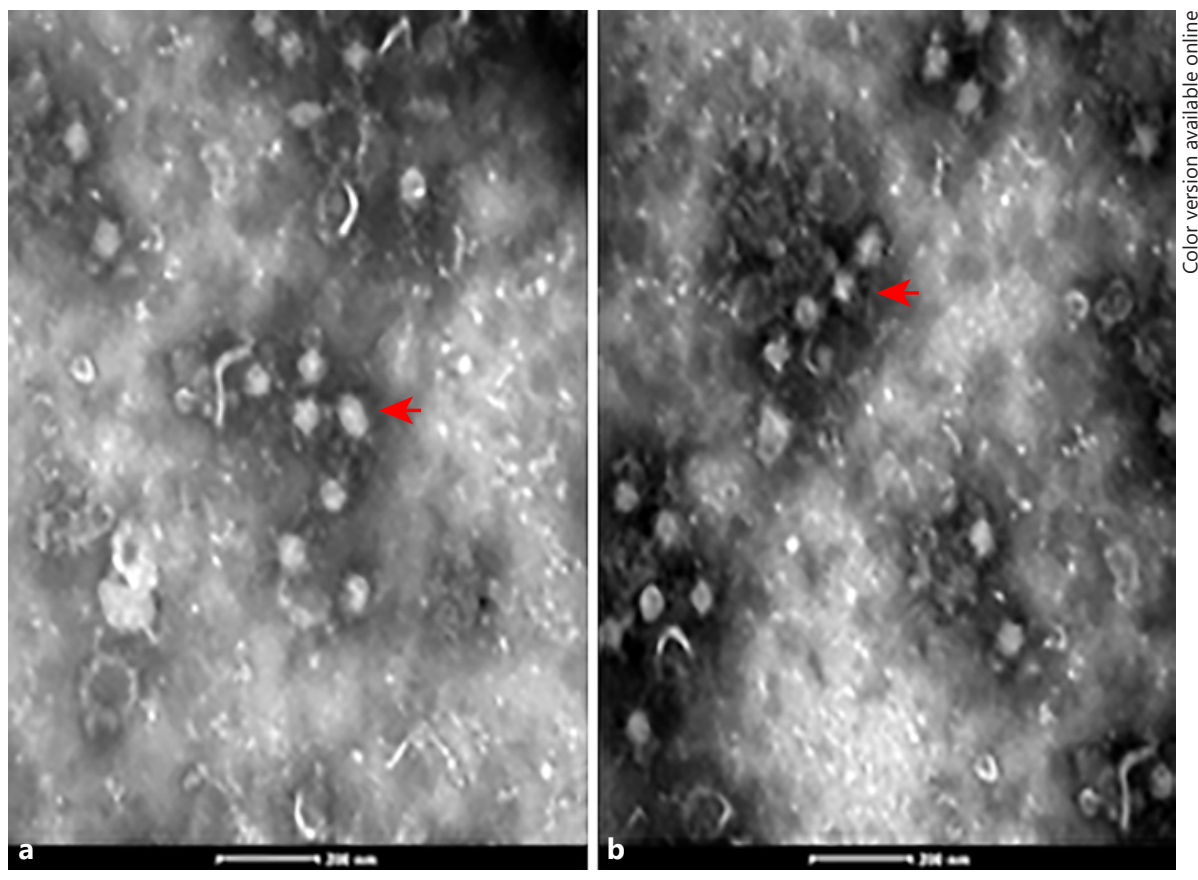

say. The cells infected with live CHIKV showed a higher number of fluorescence-positive cells as compared to the inactivated virus-treated cells. The presence of homogeneity of fluorescence in the CHIKV-infected cells suggested that the CHIKV was replicating in the cells. When a dose-dependent analysis was carried out, the cells treated with virus inactivated by UV doses less than $0.09 \mathrm{~J} / \mathrm{cm}^{2}$ showed speckled fluorescence. No signals were observed in cells treated with UV dose of $0.09 \mathrm{~J} / \mathrm{cm}^{2}$ or above (Fig. 4).

\section{Discussion}

Our results highlight that a UV dose of $0.09 \mathrm{~J} / \mathrm{cm}^{2}$ was sufficient to inactivate the Asian strain of CHIKV. The $\mathrm{UV}$-inactivated virus is replication deficient and the virus lost its ability to induce a cytopathic effect in the cells. A previous study in which CHIKV was inactivated with a combination of INA and UV also showed the same results. We tried the same dose of UV $\left(0.09 \mathrm{~J} / \mathrm{cm}^{2}\right)$ for inactivating the CHIKVECSA strain and observed similar results (data not shown). Therefore, this method could be useful to inactivate other strains of CHIKV. Reports on the inactivation of CHIKV using different chemical and physical methods are available from the early 1960s [12]. A combination of UV irradiation and formalin inactivation of CHIKV was shown to be effective in stimulating monkeys to produce anti-CHIK-neutralizing antibodies. They also reported that the immunogenicity of the combination to be higher than formalin-inactivated virus [16].

The Western blot results suggested that the structural epitopes of the UV-inactivated virus were unaltered. In the INA inactivation of CHIKV, the authors showed a reduced binding capacity of the virus to the antibodies [15]. EM analysis also showed that the virus inactivation did not affect the integrity of the virus.

IFA is considered the gold standard for the detection of CHIKV viral infection [19]. The viruses inactivated with a UV dose below $0.09 \mathrm{~J} / \mathrm{cm}^{2}$ showed speckled fluorescence in the cells, suggesting low level replication and incomplete inactivation of the pathogen. The absence of signals in cells treated with $0.09 \mathrm{~J} / \mathrm{cm}^{2}$ implied no replication and the complete inactivation of the virus. The results agreed with Sharma et al. [15], who reported that INA- and UV-inactivated CHIKV cells showed no replication.

In conclusion, UV inactivation with the reported dose could be an effective and simple inactivation method to inactivate CHIKV. It is very convenient and takes less than $2 \mathrm{~min}$ to inactivate the virus completely. Also, this inactivation method could be extrapolated to inactivate other Alphaviruses or even further standardized for serological assays to inactivate serum samples (for PRNT). More studies are needed to validate this claim. 


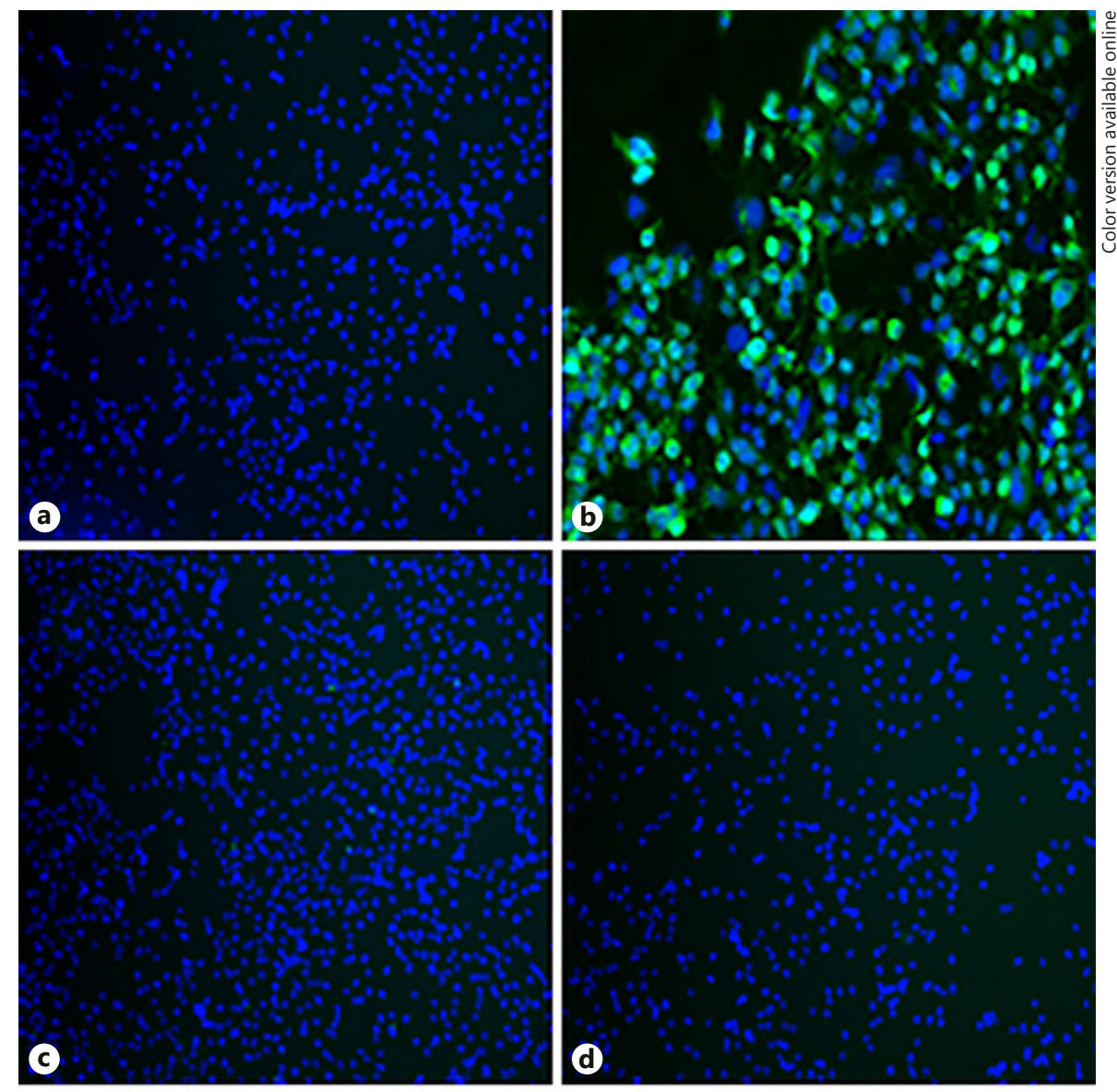

Fig. 4. Immunofluorescence analysis of RD cells treated with different doses of UV-inactivated CHIKV $\left(0.08-0.1 \mathrm{~J} / \mathrm{cm}^{2}\right)$. After $24 \mathrm{~h}$ postinfection the cells were subjected to immunostaining using in house-generated anti-CHIKV monoclonal antibodies. c RD cells treated with UV-inactivated virus (dose $<0.09 \mathrm{~J} / \mathrm{cm}^{2}$ ) showing speckled fluorescence. d, e Cells treated with inactivated (dose $0.09 \mathrm{~J} / \mathrm{cm}^{2}$ and above) CHIKV showing no signal. Untreated RD cells (a) were used as a control and RD cells infected with wild-type CHIKV (b) were used as a positive control.

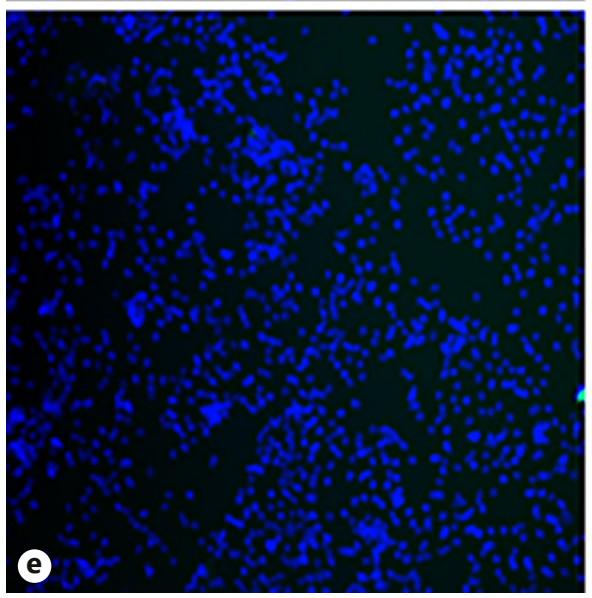

\section{Acknowledgements}

We are grateful to the Director of NIV for all the support. The authors thank Dr. A.B. Sudeep for providing the virus, Dr. G. Damle and Dr. G.N. Sapkal for the antibodies, and Dr. Atanu Basu for the EM analysis. Ms. Asha Maria Mathew is grateful to the Department of Science and Technology (DST), India, as she is supported by their DST INSPIRE Fellowship. The authors thank all the members of the NIV Kerala unit for their cooperation.

\section{Disclosure Statement}

The authors declare that they have no conflicts of interest.
Mathew/Mun/Balakrishnan 


\section{References}

1 Sourisseau M, Schilte C, Casartelli N, Trouillet C, Guivel-Benhassine F, et al: Characterization of reemerging chikungunya virus. PLoS Pathog 2007;3:e89.

2 Powers AM, Brault AC, Tesh RB, Weaver SC: Re-emergence of Chikungunya and O'nyongnyong viruses: evidence for distinct geographical lineages and distant evolutionary relationships. J Gen Virol 2000;81:471-479.

3 Sharma A, Balakathiresan NS, Maheshwari RK: Chikungunya virus infection alters expression of microRNAs involved in cellular proliferation, immune response and apoptosis. Intervirology 2015;58:332-341.

4 Burt FJ, Rolph MS, Rulli NE, Mahalingam S, Heise MT: Chikungunya: a re-emerging virus. Lancet 2012;379:662-671.

5 Kuhn RJ: Togaviridae: The Viruses and Their Replication. Philadelphia, Lippincott, Williams and Wilkins, 2007, pp. 1001-1022.

6 Johnson BW, Russell BJ, Goodman CH: Laboratory diagnosis of chikungunya virus infections and commercial sources for diagnostic assays. J Infect Dis 2016;214(suppl 5):S471S474.

7 Rauth AM: The physical state of viral nucleic acid and the sensitivity of viruses to ultraviolet light. Biophys J 1965;5:257-273.
8 Bishop JM, Quintrell N, Koch G: Poliovirus double-stranded RNA: inactivation by ultraviolet light. J Molec Biol 1967;24:125.

9 Lytle CD, Sagripanti J-L: Predicted inactivation of viruses of relevance to biodefense by solar radiation. J Virol 2005;79:14244-14252.

10 Huang YJ, Hsu WW, Higgs S, Vanlandingham DL: Temperature tolerance and inactivation of chikungunya virus. Vector Borne Zoonotic Dis 2015;15:674-677.

11 Park SL, Huang YJ, Hsu WW, et al: Virus-specific thermostability and heat inactivation profiles of alphaviruses. J Virol Methods 2016;234:152-155.

12 Wigginton KR, Pecson BM, Sigstam T, Bosshard F, Kohn T: Virus inactivation mechanisms: impact of disinfectants on virus function and structural integrity. Environ Sci Technol 2012;46:12069-12078.

13 Harrison VR, Eckels KH, Bartelloni PJ, Hampton C: Production and evaluation of a formalin-killed chikungunya vaccine. J Immunol 1971;107:643-647.
14 Kumar M, Sudeep AB, Arankalle VA: Evaluation of recombinant E2 protein-based and whole-virus inactivated candidate vaccines against chikungunya virus. Vaccine 2012;30: 6142-6149.

15 Sharma A, Gupta P, Maheshwari RK: Inactivation of chikungunya virus by 1,5 iodonapthylazide. Virol J 2012;9:301.

16 Nakao E, Hotta S: Immunogenicity of purified, inactivated chikungunya virus in monkeys. Bull World Health Organ 1973;48:559562.

17 Tiwari M, Parida M, Santhosh SR, Khan M, Dash PK, Rao PV: Assessment of immunogenic potential of Vero adapted formalin inactivated vaccine derived from novel ECSA genotype of chikungunya virus. Vaccine 2009;27:2513-2522.

18 Sharma A, Raviv Y, Puri A, Viard M, Blumenthal R, Maheshwari RK: Complete inactivation of Venezuelan equine encephalitis virus by 1,5-iodonaphthylazide. Biochem Biophys Res Commun 2007;358:392-398.

19 Yap G, Pok KY, Lai YL, Hapuarachchi HC, Chow A, Leo YS, Tan LK, Ng LC: Evaluation of chikungunya diagnostic assays: differences in sensitivity of serology assays in two independent outbreaks. PLoS Negl Trop Dis 2012; 4:e753. 